\title{
Response functions and cross sections for inclusive neutrino scattering off ${ }^{2} \mathrm{H},{ }^{3} \mathrm{H}$ and ${ }^{3} \mathrm{He}$
}

\author{
Alessandro Grassi ${ }^{1 \star}$, Jacek Golak ${ }^{1}$, Roman Skibiński ${ }^{1}$, Kacper Topolnicki ${ }^{1}$, \\ Henryk Witała ${ }^{1}$, Hiroyuki Kamada ${ }^{2}$ and Laura Elisa Marcucci ${ }^{3}$
}

1 M. Smoluchowski Institute of Physics, Jagiellonian University, PL-30348 Cracow, Poland

2 Department of Physics, Faculty of Engeneering,

Kyushu Institute of Technology, Kitakyushu 804-8550, Japan

3 Department of Physics, University of Pisa, IT-56127 Pisa,

Italy and INFN-Pisa, IT-56127 Pisa, Italy

$\star$ alessandro.grassi@doctoral.uj.edu.pl

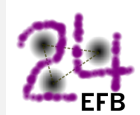

Proceedings for the 24th edition of European Few Body Conference,

Surrey, UK, 2-6 September 2019

doi:10.21468/SciPostPhysProc.3

\begin{abstract}
For several decades there has been an interest in studying neutrino scattering on light nuclei, with the hope of increasing knowledge about the theory of weak interactions and electroweak unification. A study of several neutrino induced reactions on light nuclei $\left({ }^{2} \mathrm{H},{ }^{3} \mathrm{H}\right.$ and $\left.{ }^{3} \mathrm{He}\right)$ is presented here. The cross section for these reactions is evaluated using the nuclear response functions, which holds the information about the nuclear interactions and structure. This approach has been tested for break-up reactions on the deuteron before and is now extended to three-nucleon break-up reactions.
\end{abstract}

(c) (1) Copyright A. Grassi et al.

This work is licensed under the Creative Commons

Attribution 4.0 International License.

Published by the SciPost Foundation.
Received 07-11-2019

Accepted 10-12-2019

Published 26-02-2020

Check for updates

\section{Introduction}

The interest in neutrino reactions on nuclei lies not only in nuclear physics, but also in other fields such as astrophysics and particle physics. Moreover, the construction of neutrino detectors requires knowledge about these types of reactions $[1,2]$. Calculations performed in both coordinate and momentum space have given equivalent results, suggesting that at low energies the theoretical uncertainty may be small. These studies were performed by adopting semi-phenomenological potentials as well as ones based on chiral effective field theory [3], and the related single and two nucleon current operators.

Here we present a new contribution to this field. The ingredients of this study are the semi-phenomenological potential AV18 [4] and the single nucleon current. 


\section{The differential cross section}

The differential cross section depends on the transition matrix element of the process $M_{f i}$. It appears in its quadratic form, i.e.

$$
\left|M_{f i}\right|^{2}=\left|L_{\lambda} N^{\lambda}\right|^{2}
$$

where the $L_{\lambda}\left(N_{\lambda}\right)$ are the transition matrix elements for the leptonic (hadronic) current.

By calling $\mathbf{k}\left(\mathbf{k}^{\prime}\right)$ the initial (final) lepton momentum, and by choosing a reference frame in which the transferred momentum $\mathbf{Q}=\mathbf{k}-\mathbf{k}^{\prime}$ is parallel to the $z$-axis we can identify the parts in $\left|M_{f i}\right|^{2}$ which contribute in the case of inclusive reactions, where only the final lepton is detected and write Eq. (1) as

$$
\left|M_{f i}\right|^{2}=V_{00}\left|N^{0}\right|^{2}+V_{P P}\left|N_{+1}\right|^{2}+V_{M M}\left|N_{-1}\right|^{2}+V_{Z Z}\left|N_{z}\right|^{2}+V_{Z 0} \operatorname{Re}\left(2 N^{0} N_{z}^{*}\right) .
$$

Here the $V_{i j}$ functions are combinations of the $L_{\lambda} L_{\lambda^{\prime}}{ }^{*}$ functions [5].

The inclusive response functions are defined as

$$
R_{A B}^{i n c}=\sum_{m_{i} m_{f}} \int \mathrm{d} f \delta\left(E_{C M}-E_{f}\right)\left\langle\Psi_{f}\left|j^{A}\right| \Psi_{i}\right\rangle\left\langle\Psi_{i}\left|j^{B}\right| \Psi_{f}\right\rangle,
$$

where $\left|\Psi_{i}\right\rangle\left(\left|\Psi_{f}\right\rangle\right)$ is the initial (final) nuclear state and $j^{A(B)}$ are single-nucleon hadronic current operators. By defining

$$
N^{A} \equiv\left\langle\Psi_{f}\left|j^{A}\right| \Psi_{i}\right\rangle
$$

we can express the differential cross section for this kind of reactions as [5]

$$
\frac{d^{3} \sigma}{d E^{\prime} d \Omega^{\prime}}=\frac{G_{F}^{2} \cos ^{2} \theta_{C}}{(2 \pi)^{2}} F\left(Z, E^{\prime}\right) \frac{k^{\prime}}{8 E}\left(V_{00} R_{00}+V_{P P} R_{P P}+V_{M M} R_{M M}+V_{Z Z} R_{Z Z}+V_{Z 0} R_{Z 0}\right),
$$

where $G_{F}$ is the Fermi constant, $\theta_{C}$ is the Cabbibo angle, $k^{\prime}\left(E^{\prime}\right)$ is the final lepton momentum magnitude (energy), $E$ is the initial lepton energy, $Z$ is the final charge of the hadronic state and $F\left(Z, E^{\prime}\right)$ is the Fermi function which takes into account the Coulomb distortion of the final electron wave function. For neutral current reactions $\cos \theta_{C}$ and $F\left(Z, E^{\prime}\right)$ are substituted by 1 . The Fermi function is defined as

$$
F\left(Z, E^{\prime}\right)=2(\gamma+1)\left(2 k^{\prime}\right)^{2 \gamma-2}\left|\frac{\Gamma(\gamma-i y)}{\Gamma(1+2 \gamma)}\right|^{2},
$$

with $\gamma$ and $y$ as

$$
\gamma=\sqrt{1-Z \alpha} \quad y=Z \alpha \frac{E^{\prime}}{k^{\prime}},
$$

where $\alpha$ is the fine structure constant.

In order to evaluate the differential cross section in Eq. (5), it is therefore necessary to calculate the previously defined response functions. These functions depend only on the transferred momentum $Q$ and on the final internal hadronic kinetic energy $E_{C M}$.

\section{Results}

We tested the response function method by comparing the results obtained for the deuteron break-up reactions with direct calculations. We found less than one percent deviations between 

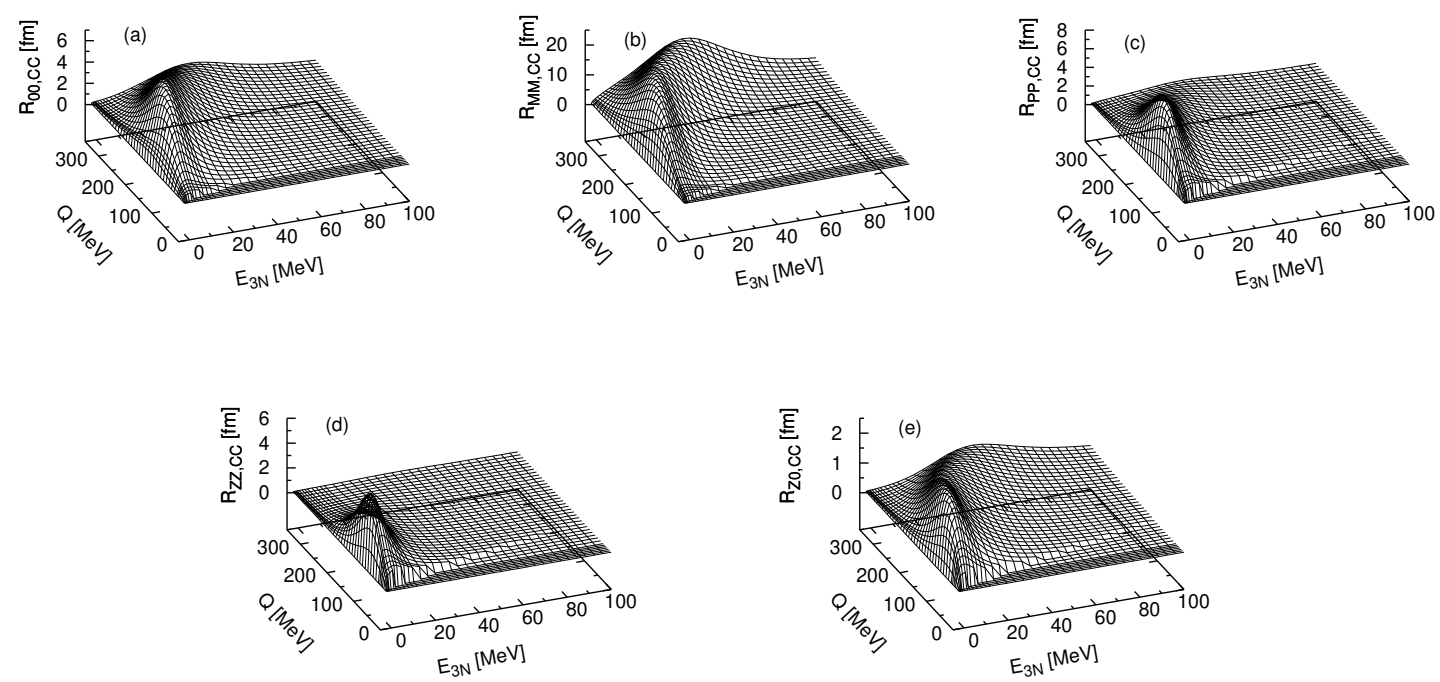

Figure 1: The response functions for the charged current reactions on ${ }^{3} \mathrm{He}$ as a function of the internal hadronic energy in for the $3 \mathrm{~N}$ system $E_{3 N}$ and the transferred momentum $Q$. The figure has been taken from Ref. [6].

the two methods and then proceeded to investigate $3 \mathrm{~N}$ break-up reactions using the response function scheme.

We evaluated the response functions for these reactions in four grids, each of them containing circa 2000 points in the $\left(E_{C M}, Q\right)$ space. The results for the antineutrino break-up on ${ }^{3} \mathrm{He}$ are shown in Fig. 1. Similar results were obtained for the other three grids.

Having these functions it is then possible to calculate the differential cross section and the total cross section at a given energy,

$$
\sigma(E)=\int_{0}^{\pi} \mathrm{d} \theta^{\prime} \sin \theta^{\prime} \int_{0}^{2 \pi} \mathrm{d} \phi^{\prime} \int \mathrm{d} E^{\prime} \frac{d^{2} \sigma}{d E^{\prime} d \Omega^{\prime}} .
$$

The results for the total cross section are shown in Fig. 2.

\section{Conclusions}

We performed a study of the differential and total cross sections for several (anti) neutrino break-up reactions on ${ }^{3} \mathrm{H}$ and ${ }^{3} \mathrm{He}$. The method used has been tested in the simpler break-up reaction on ${ }^{2} \mathrm{H}$, for which we could adopt a direct method to check the convergence of the results.

This study has been performed by using the simple single-nucleon current operator and can be improved by adding many-nucleon currents.

\section{Acknowledgements}

This work is a part of the LENPIC project. The numerical calculations were partially performed on the supercomputer cluster of the JSC, Jülich, Germany. 

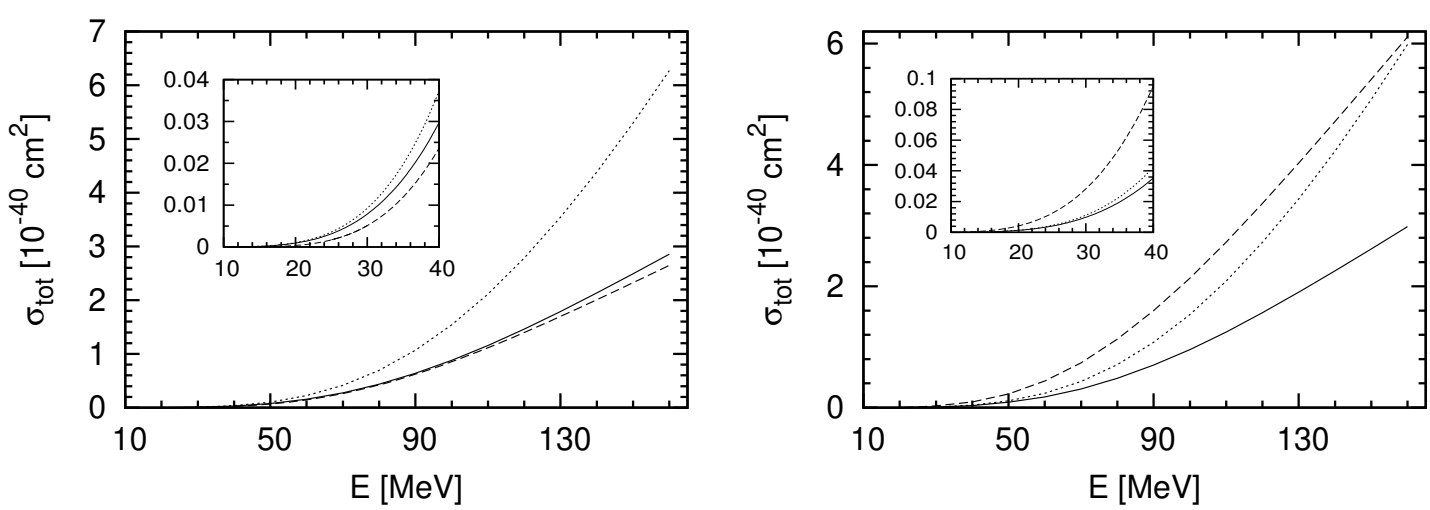

Figure 2: (left) Cross section for three inclusive (anti)neutrino reactions with ${ }^{3} \mathrm{H}$ : charged current electron antineutrino disintegration of ${ }^{3} \mathrm{H}$ (dashed line), neutral current electron antineutrino disintegration of ${ }^{3} \mathrm{H}$ (solid line), neutral current electron neutrino disintegration of ${ }^{3} \mathrm{H}$ (dotted line). (right) Same as left, but substituting ${ }^{3} \mathrm{H}$ with ${ }^{3} \mathrm{He}$. These figures have been taken from Ref. [6].

Funding information This work was supported by the Polish National Science Centre under Grants No. 2016/22/M/ST2/00173 and 2016/21/D/ST2/01120 as well as by BMBF (contract No. 05P18PCFP1) and by DFG and NSFC (TRR 110).

\section{References}

[1] S. Nakamura, T. Sato, V. Gudkov and K. Kubodera, Neutrino reactions on the deuteron, Phys. Rev. C 63, 034617 (2001), doi:10.1103/PhysRevC.63.034617.

[2] S. Nakamura, T. Sato, S. Ando, T.-S. Park, F. Myhrer, V. Gudkov and K. Kubodera, Neutrino-deuteron reactions at solar neutrino energies, Nucl. Phys. A 707, 561 (2002), doi:10.1016/S0375-9474(02)00993-4.

[3] M. Butler, J.-W. Chen and X. Kong, Neutrino-deuteron scattering in effective field theory at next-to-next-to-leading order, Phys. Rev. C 63, 035501 (2001), doi:10.1103/PhysRevC.63.035501.

[4] R. B. Wiringa, V. G. J. Stoks and R. Schiavilla, Accurate nucleon-nucleon potential with charge-independence breaking, Phys. Rev. C 51, 38 (1995), doi:10.1103/PhysRevC.51.38.

[5] J. Golak, R. Skibiński, K. Topolnicki, H. Witała, A. Grassi, H. Kamada and L. E. Marcucci, Momentum space treatment of inclusive neutrino scattering off the deuteron and trinucleons, Phys. Rev. C 98, 015501 (2018), doi:10.1103/PhysRevC.98.015501.

[6] J. Golak, R. Skibiński, K. Topolnicki, H. Witała, A. Grassi, H. Kamada and L. E. Marcucci, From response functions to cross sections in neutrino scattering off the deuteron and trinucleons, Phys. Rev. C 100, 064003 (2019), doi:10.1103/PhysRevC.100.064003. 